\title{
The STAR CENTRE at Sheffield Hallam University
}

\author{
By John H Parkinson, D. Ashton, K. Atkin \& B. Harrison
}

School of Science and Mathematics, Sheffield Hallam University, Pond Street, Sheffield S1 1WB

\section{Introduction}

The Star Centre is a national astronomy and space science base which

- facilitates public access to news and information

- promotes public awareness, interest, enjoyment and understanding.

The Star Centre meets these twin aims by providing an information service which can be accessed in a variety of ways and by offering a menu of public observing events.

The concept of a national astronomy base developed as part of the Centre for Science Educations growing portfolio of initiatives in both the formal education sector and the wider umbrella of the Public Understanding of Science. In December 1996 the Star Centre was launched with the aid of a Royal Society COPUS development grant and matching funding from Sheffield Hallam University.

This paper summarises the main activities of the Star Centre, gives some impression of the public response and outlines plans for future development.

\section{The Star Centre in Context}

The Star Centre reaches out directly to schools and the general public and is part of the growing network of long-term projects at the Centre for Science Education (CSE) within the School of Science and Mathematics as shown in Figure 1. The largest of these projects is the UK Research Council funded Pupil Researcher Initiative (PRI) in which school pupils in the 14-16 age range explore science topics through research briefs. The PRI provides resources, activities, strategies and support for science teachers and their pupils so that pupils will experience the excitement and relevance of science and engineering research and so develop a lasting interest and enthusiasm. All aspects of the research process are involved and there are opportunities for Science Fairs, Pupil Researcher Conferences and Roadshows. There is a national network for recruiting University Research/Graduate students who are trained appropriately before being installed as Researchers in Residence in schools where they have opportunities to discuss their work with pupils, further develop their communication skills and help to support the science learning of pupils. The Centre acts as the co-ordination focus for the PRI projects involving astronomy.

The Star Centre also has links to the Internet-based project Schools On-Line and to the Science Alliance, which connects primary schools with industry.

Stardome is a mobile Starlab planetarium supported in part by the University which reaches a total audience of around 12,000 people per year, mostly in schools but also at public events. Schoolchildren and adults often make their first contact with the Star Centre after a Stardome experience.

Sheffield Hallam University makes a major commitment to Public Understanding of Science activities which include the annual Sheffield Science Festival, the National week of Science, Engineering and Technology, various lectures particularly around Christmas, the Yorkshire Science-a-thon and, of course, National Astronomy Week. 

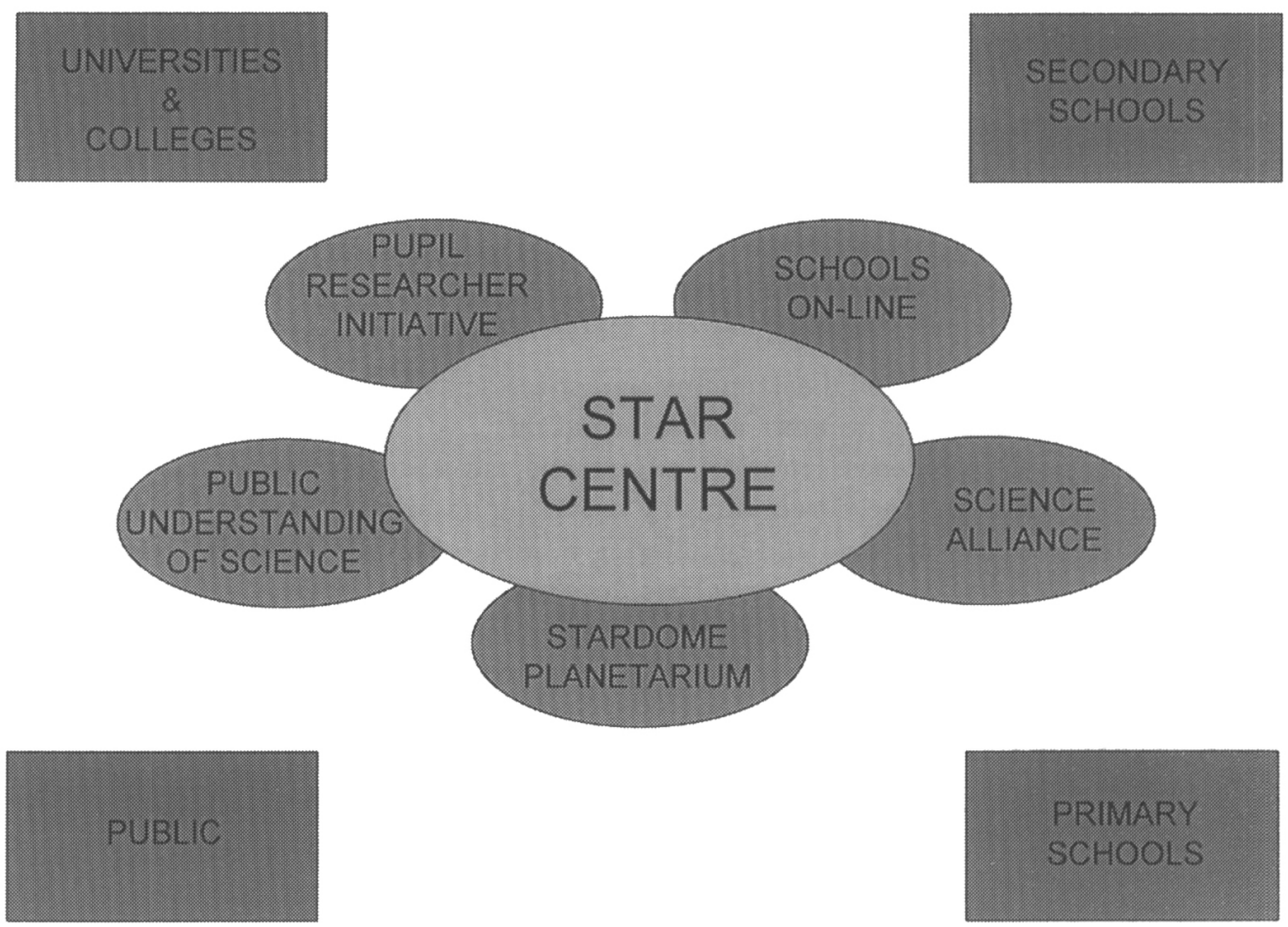

Figure 1. The network of projects within the Centre for Science Education at Sheffield Hallam University.

\section{Activities}

Star Centre activities are directed towards encouraging and enabling people to observe the night sky and to learn more about the Universe at large. The Centre aims to appeal particularly to interested sky watchers who would like to become recreational observers but we also cater for enthusiasts who wish to join an observing project. Some of the main activities are shown in Figure 2.

\subsection{The Information Centre}

Star Centre's information room is located at the University's Collegiate Crescent Campus. At the moment funds allow the base to be staffed by resident astronomers on only one day each week. Users write, phone, fax or e-mail to access information or pose questions on news, opinion, observation, equipment, events or background. The resident astronomers act as interpreters of information derived from the Internet, software, reference books and their own experience.

\subsection{Star Nights}

Public observing sessions from dark-sky sites in Derbyshire have been held on a regular basis. The two Star Nights held this year have attracted capacity audiences totalling 600 people. Unfortunately, both nights suffered typical British weather - intermittent clouds! The response, however, was very enthusiastic and visitors discovered that astronomy was more than just looking through telescopes and made use of a temporary information room, short talks, computer stations, video rooms and a cafe. 


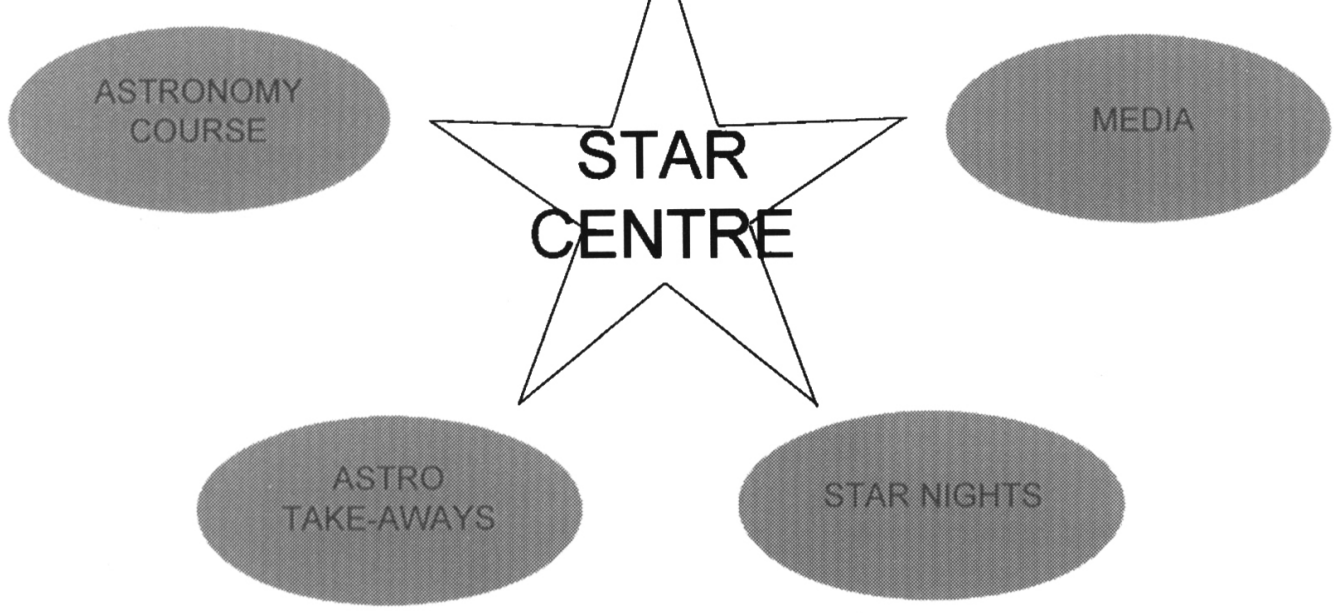

Figure 2. The major activities of the Star Centre.

\subsection{Astro Take-Aways}

These are smaller scale observing nights put on in response to requests from various interest groups. In the first half of 1996, 5 take-aways have been attended by a total of $200+$ people.

\subsection{Observing Projects}

The Centre currently supports 2 projects:-

Starwatch UK - a light pollution survey established by the Royal Greenwich Observatory, the British Astronomical Association, the Federation of Astronomical Societies and others.

Meteor Watch - a meteor observation project with results fed to Star Centre for collation and analysis.

\subsection{Astronomy Course}

The Centre sponsors a 24 week course covering observing topics. This is over-subscribed with 20 members of the general public and a waiting list!

\subsection{Stardome}

Stardome has operated since 1993 , giving 1,320 presentations to a total audience of 34,400 people in that time. The planetarium visits around 100 venues per year, $70 \%$ of which are schools. The remaining visits involve PUS activities such as events at The Science Museum London, SET Week events, Association for Science Education, Towards 2000 Conference, Sheffield Festival, Association for Environmental Education, Star trek Conventions etc. 


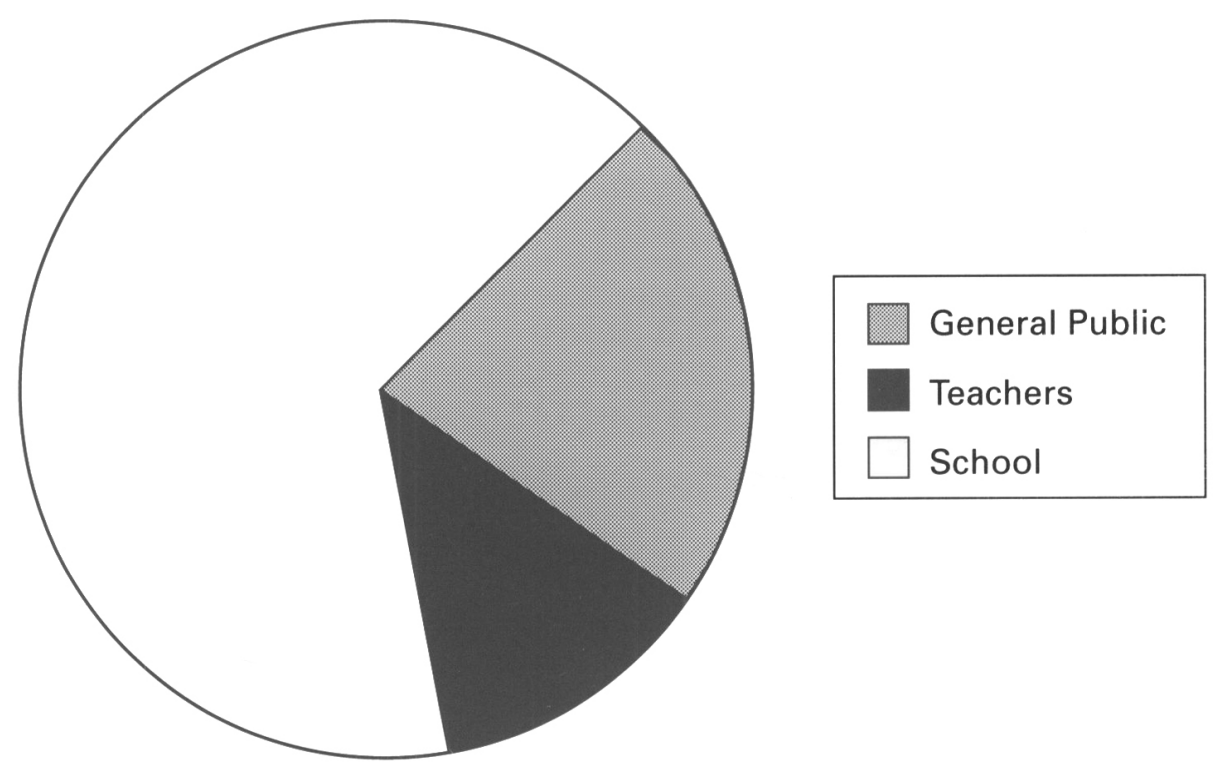

Figure 3. Analysis of the enquiries by type of enquirer.

\subsection{Media}

Star Centre provides a monthly 600 word astronomy column, for the Sheffield Telegraph, a regional newspaper, together with a regular slot on Radio Sheffield, where Star Centre astronomers explore a topic and then answer questions via a phone-in. The Centre also acts as a general enquiry point for the media at large.

\subsection{Starter Packs}

In response to user requests, Star Centre astronomers have produced a 24 page booklet for people who want to participate in recreational observation. The pack includes equipment guides, star maps and advice on observing planets and deep space objects.

\section{Usage of the Centre}

In the first half of 1996 the Star Centre received 360 enquiries. Figures 3 and 4 show how these were broken down.

Most schoolchildren request information and pictures about planets. Teachers often require classroom activities and the Star Centre supplies them using current Sheffield Hallam University educational project material.

Users will shortly be able to access Star Centre's own Internet site and the pages will reflect the aims of the Centre - to provide accessible information and encourage observation of the skies. The three themes of the site are:-

- Links with local and national organisations and events including the Bradford Robotic Telescope, societies, planetaria, observatories etc

- Observation - guides, maps, challenges, projects and equipment 


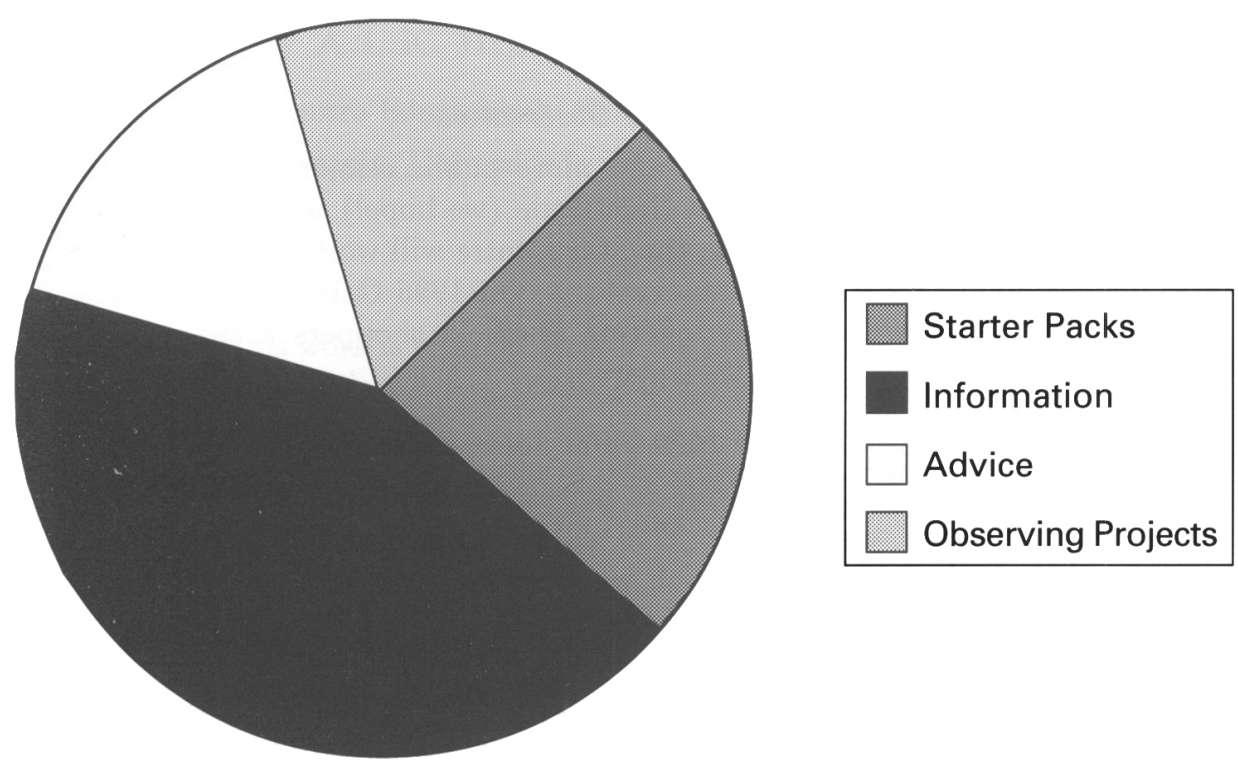

Figure 4. Analysis of enquiries by type of enquiry.

- Information and data on astronomical objects, including school activities.

The Star Centre URL is http://www.shu.ac.uk/schools/sci/star_centre/contents.htm.

\section{Future Developments}

Further funding in the form of grants and sponsorship is being sought to develop the activities of Star Centre as we continue to enhance the national profile of the information centre, develop activities for use in schools and extend our public observation programme. The Centre will seek to raise awareness in astronomical establishments of its ability to deal with enquiries from the public. Visitors to planetaria and observatories could use the Star Centre as an ongoing and accessible follow-up to their visit.

Star Centre will gain a more substantial international profile when its Internet site comes on-line during the summer of 1996. As well as acting as an information source, the interactive nature of the contents will help the Centre establish itself as a co-ordination base and the hub of a network of observers, particularly those new to the enjoyment of recreational observation.

\subsection{Schools}

Star Centre staff are primarily astronomy educators. Utilising active learning principles which underpin many of Sheffield Hallam University's school's projects, the Centre aims to produce school-based resource packages targeted at the National Curriculum science unit Earth and Beyond at both primary and secondary levels.

Through the Science Alliance project, the Star Centre is to contribute to an astronomy day for primary schools in the spring of 1997 . This will be a pilot for further one-day 
conferences for schoolchildren at different levels, including those choosing the A-level Physics astronomy option.

Young astronomers will be targeted in the Astronomy Suitcase which the Star Centre has been asked to produce for Scout and Guide groups. As well as helping youngsters gain their astronomy badge, the suitcases will provide a model for practical-based resource packages for schools.

\subsection{Public Observation}

Star Nights have proved very popular and more public sessions are planned. These will be themed nights featuring Comet Hale-Bopp (we hope!) and the Geminid meteor shower. In National Astronomy Week, the Star Centre will work with other local groups to offer five observing nights at an observatory. On 1996 October 12, the Star Centre astronomers will take to the streets, offering Sheffield shoppers a chance to view the partial solar eclipse from a city centre square.

\subsection{The 1999 Total Solar Eclipse}

The Star Centre will play a major role in increasing awareness and safe enjoyment of the 1999 August 11 total solar eclipse. This will be the first such eclipse visible from the UK mainland since the 1926 event which was very badly affected by weather. Totality only occurs in Cornwall but the whole of the British Isles will experience a major partial eclipse. It is hoped to extend the PRI framework to place suitable material in every school in the country enabling students of all ages to share the excitement of carrying out scientific investigations of this cosmic event. Expeditions from schools around the country will be organised to observe totality from Cornwall.

\section{Summary}

The Star Centre has established a firm local presence and has become an integral part of the continuing educational and Public Understanding projects initiated at Sheffield Hallam University. In the future, an increased profile will involve public participation through the Internet and contact with the information centre, together with educational projects to enhance interest and learning in schools.

Underlying all the developments is the philosophy that the fascination of astronomy - new discoveries, different worlds, bizarre objects, cosmic evolution, enjoyment of the night sky - should be made easily accessible to people of all ages. 